\title{
Optimal regularity in the optimal switching problem
}

\author{
Gohar Aleksanyan \\ Received 7 October 2014; received in revised form 12 June 2015; accepted 25 June 2015
}

Available online 23 July 2015

\begin{abstract}
In this article we study the optimal regularity for solutions to the following weakly coupled system with interconnected obstacles

$$
\left\{\begin{array}{l}
\min \left(-\Delta u^{1}+f^{1}, u^{1}-u^{2}+\psi^{1}\right)=0 \\
\min \left(-\Delta u^{2}+f^{2}, u^{2}-u^{1}+\psi^{2}\right)=0,
\end{array}\right.
$$

arising in the optimal switching problem with two modes.

We derive the optimal $C^{1,1}$-regularity for the minimal solution under the assumption that the zero loop set $\mathscr{L}:=\left\{\psi^{1}+\psi^{2}=0\right\}$ is the closure of its interior. This result is optimal and we provide a counterexample showing that the $C^{1,1}$-regularity does not hold without the assumption $\mathscr{L}=\overline{\mathscr{L}^{0}}$.

๑ 2015 L'Association Publications de l'Institut Henri Poincaré. Published by Elsevier B.V. All rights reserved.
\end{abstract}

Keywords: Optimal switching problem; Regularity theory; The obstacle problem; Double obstacle problem; Free boundary problem; Nonlinear elliptic system

\section{Introduction}

We consider the following system of weakly coupled equations of obstacle type

$$
\left\{\begin{array}{l}
\min \left(-\Delta u^{1}+f^{1}, u^{1}-u^{2}+\psi^{1}\right)=0 \\
\min \left(-\Delta u^{2}+f^{2}, u^{2}-u^{1}+\psi^{2}\right)=0,
\end{array}\right.
$$

with given Dirichlet boundary conditions $u^{i}=g^{i}$ on $\partial \Omega$. These type of systems arise in optimal switching problems with two switching modes. Here $f^{1}$ and $f^{2}$ are the running cost functions corresponding to the switching modes. The functions $\psi^{1}$ and $\psi^{2}$ are the costs of switching from one mode to the other. More details on the optimal switching problem are provided in Section 2.1.

The uniqueness and $C^{1,1}$-regularity of the solutions to such systems have been studied in the literature under the assumption that the switching costs are nonnegative constants, [4,7,2]. Obstacle type weakly coupled systems with first order Hamiltonians and nonconstant switching costs have been studied in [3,6]. In the paper [3], Section 5 the authors investigate the speed of convergence of the solutions to a penalized system, they also show that the solution

\footnotetext{
E-mail address: galeksan@kth.se.
} 
of the first order Hamilton-Jacobi obstacle type system is Lipschitz continuous, under the assumption that each of the switching costs is bounded from below by a positive constant.

In our paper we make only the nonnegative loop assumption. This is a necessary condition for the system to be well-defined. Indeed, let $\left(u^{1}, u^{2}\right)$ be a solution to (1), then $u^{1}-u^{2}+\psi^{1} \geq 0$ and $u^{2}-u^{1}+\psi^{2} \geq 0$, which implies

$$
\psi^{1}(x)+\psi^{2}(x) \geq 0 .
$$

In the optimal switching setting, the condition (2) prevents the agent from making arbitrary gains by looping, in the sense that $\psi^{1}(x)+\psi^{2}(x)$ is the cost of switching from one mode to the other and immediately switching back. We denote the set where it is possible to switch for free by

$$
\mathscr{L}=\left\{x \in \Omega \mid \psi^{1}(x)+\psi^{2}(x)=0\right\},
$$

and call it free switching or zero loop set.

By using the penalization/regularization method we derive the existence of solutions, showing that through a subsequence the solutions of the penalized system converge to the minimal solution $\left(u_{0}^{1}, u_{0}^{2}\right)$ to (1). Then we see that the solution $u_{0}^{i} \in C^{1, \gamma}$, for every $0<\gamma<1$ and

$$
\left\|\Delta u_{0}^{i}\right\|_{L^{\infty}(\Omega)} \leq \max _{i}\left\|\Delta \psi^{i}\right\|_{L^{\infty}(\Omega)}+3 \max _{i}\left\|f^{i}\right\|_{L^{\infty}(\Omega)} .
$$

The aim of the paper is to investigate if the solutions are $C^{1,1}$, which is the best regularity that we can hope that the solutions achieve. The structure of our system shows that at some subdomains of $\Omega$, the regularity of the solutions can be derived by already known $C^{1,1}$-regularity results for the obstacle problem. In our discussion we see that the main point is to describe the regularity at so called meeting points lying on $\partial \mathscr{L}$, the boundary of the zero loop set.

In the main theorem, Theorem 4, we show that at the meeting points $x_{0} \in \partial \mathscr{L}^{0} \cap \Omega$ the solutions are $C^{2, \alpha}$, under the assumption that $f^{i} \in C^{\alpha}$ and $\psi^{i} \in C^{2, \alpha}$. By $\mathscr{L}^{0}$ we denote the interior of the set $\mathscr{L}$, and by pointwise $C^{2, \alpha}$ regularity we mean uniform approximation with a second order polynomial with the speed $r^{2+\alpha}$.

The idea of the proof is the same as in deriving the optimal regularity for the no-sign obstacle problem in [1]. The proof is based on the $B M O$-estimates for $D^{2} u_{0}^{1}$ and $D^{2} u_{0}^{2}$ following from the estimate (3). At the point $x_{0}$, we consider $r^{2+\alpha}$-th order rescalings of $u_{0}^{i}$ denoted by $v_{r}^{i}$, and show that these are uniformly bounded in $W^{2,2}\left(B_{1}\right)$. Then, looking at the corresponding system for $\left(v_{r}^{1}, v_{r}^{2}\right)$, we conclude that the rescalings are uniformly bounded in the ball $B_{1}$.

In the end we justify our assumption $0 \in \partial \mathscr{L}^{0}$ with a counterexample: We consider a particular system in $\mathbb{R}^{2}$, where the zero loop set $\mathscr{L}=\{0\}$, then we find an explicit solution, that is not $C^{1,1}$.

The paper is structured as follows: In Section 2 we provide some background material. In Section 3 we use the penalization method to derive the existence of strong solutions, and observe that these are actually minimal solutions. The main results are presented in the last section, where we prove that the minimal solution is locally $C^{1,1}$ if the zero loop set is the closure of its interior, and provide a counterexample to $C^{1,1}$-regularity when $\psi^{1}+\psi^{2}$ has an isolated zero.

\section{Background material}

In this section we state some known results, which we use in our discussion, without giving any proofs.

\subsection{Optimal switching problem}

Let $\Omega \subset \mathbb{R}^{n}$ be a bounded domain with a smooth boundary. We consider an agent that can be anywhere in $\Omega$ and in one of a finite number $m$ of states. For every $1 \leq i \leq m$, the agent moves in $\Omega$ according to a diffusion

$$
d x=b_{i}(x) d t+\sigma_{i}(x) d W_{t},
$$

where $W_{t}$ is a Brownian motion in a suitable probability space, $b_{i}: \Omega \rightarrow \mathbb{R}^{n}$ and $\sigma_{i}: \Omega \rightarrow \mathbb{R}^{n \times m}$ are smooth functions. The generator of the diffusions is denoted by $L^{i} v=\frac{1}{2} \sigma_{i} \sigma_{i}^{T}: D^{2} v+b_{i} \cdot D v$.

The agent can switch from any diffusion mode to another. At every instant $t$ the agent pays a running $\operatorname{cost} f^{i(t)}(x)$, depending on the present state $i(t)$ and position $x$. Additionally, when changing state $i$ to state $j$ he incurs in a switching cost $-\psi^{i j}(x)$. Finally, when the diffusion reaches the boundary and the agent is in state $i$, the process is stopped 
and a cost $-g^{i}(x)$ is incurred. As it is traditional in optimal switching setting, we consider the problem of maximizing a certain profit (the negative of the cost) functional

$$
\begin{aligned}
u_{i}(x)= & \max _{i(t), i(0)=i} E\left[\int_{0}^{T_{\partial \Omega}} f_{i(t)}(x(t)) d t\right. \\
& -\sum_{t \leq T_{\partial \Omega}} \psi_{i\left(t^{-}\right), i\left(t^{+}\right)}(x(t))+g_{i\left(T_{\partial \Omega}\right)}\left(x\left(T_{\partial \Omega}\right)\right],
\end{aligned}
$$

where $T_{\partial \Omega}$ denotes the exit time of $\Omega$. Additionally, the convention $\psi_{i i}=0$ is assumed.

As it has been discussed in the literature [7,2], the corresponding value function $u^{i}$ solves the following system:

$$
\min _{i}\left(-L^{i} u^{i}+f^{i}, \min _{j}\left(u^{i}-u^{j}+\psi^{i j}\right)\right)=0
$$

with boundary conditions $u^{i}=g^{i}$ on $\partial \Omega$.

For the optimal switching problem to be well defined, we need to impose the nonnegative loop condition: Let $i_{0}, i_{1}, \ldots, i_{l}=i_{0}$ be any loop of length $l$, i.e. including $l$ number of states. Assume that $\left(u^{1}, u^{2}, \ldots, u^{m}\right)$ is a solution to system (4), then $u^{i}-u^{j}+\psi^{i j} \geq 0$ for any $i, j \in\{1,2, \ldots, m\}$, then after summing the equations over the loop, we get

$$
\sum_{j=1}^{l} \psi_{i_{j-1}, i_{j}} \geq 0
$$

This condition is a necessary assumption for the existence of a solution to (3), and it prevents the agent from making arbitrary gains by looping.

In this paper we consider a system, arising in a model optimal switching problem with only two states.

\subsection{The Poisson equation, Calderon-Zygmund estimates}

We start by recalling the definition of the Hölder space $C^{k, \gamma}$. Let us denote the continuity norm

$$
\|u\|_{C(\bar{\Omega})}=\sup _{x \in \Omega}|u(x)|,
$$

and the Hölder seminorm

$$
[u]_{C^{0, \gamma}(\bar{\Omega})}=\sup _{x, y \in \Omega, x \neq y} \frac{|u(x)-u(y)|}{|x-y|^{\gamma}} .
$$

Definition 1. The Hölder space $C^{k, \gamma}(\Omega)$ consists of all functions $u \in C^{k}(\bar{\Omega})$ such that

$$
\|u\|_{C^{k, \gamma}(\Omega)}:=\sum_{|\alpha| \leq k}\left\|D^{\alpha} u\right\|_{C(\bar{\Omega})}+\sum_{|\alpha|=k}\left[D^{\alpha} u\right]_{C^{0, \gamma}(\bar{\Omega})}<\infty .
$$

The next theorem states the known regularity of the solutions to the Poisson equation $\Delta u=f$, under the assumption that $f$ is Hölder continuous, and can be found in the book [5].

Theorem 1. Assume that $f \in C^{\gamma}$, then there exists a classical solution to the Poisson equation

$$
\Delta u=f \text { in } \Omega .
$$

Moreover, the solution is locally $C^{2, \gamma}(\Omega)$, and for every $\Omega^{\prime} \Subset \Omega$

$$
\|u\|_{C^{2, \gamma}\left(\Omega^{\prime}\right)} \leq C_{n, \gamma}\left(\Omega^{\prime}\right)\left(\|u\|_{C(\bar{\Omega})}+\|f\|_{C^{0, \gamma}(\Omega)}\right),
$$

where the constant $C_{n, \gamma}\left(\Omega^{\prime}\right)$ depends on $\operatorname{diam} \Omega^{\prime}$ and $\operatorname{dist}\left(\Omega^{\prime}, \partial \Omega\right)$. 
Next let us recall the definition of $B M O$ spaces, and then state the Calderon-Zygmund estimates for the Poisson equation $\Delta u=f$, when $f \in L^{p}, 1<p \leq \infty$.

Definition 2. We say that a function $u \in L^{2}(\Omega)$ is in $B M O(\Omega)$ if

$$
\|f\|_{B M O(\Omega)}^{2}:=\sup _{x \in U, r>0} \frac{1}{r^{n}} \int_{B_{r}(x) \cap \Omega}\left|f(y)-(f)_{r, x}\right|^{2} d y+\|f\|_{L^{2}(\Omega)}^{2}<\infty,
$$

where $(f)_{r, x}$ is the average of $f$ in $B_{r}(x) \cap \Omega$.

The proofs of the following results can be found in [5] when $p<\infty$ and in [9] when $p=\infty$.

\section{Theorem 2. Consider the equation}

$$
\Delta u=f \text { in } B_{2 R} .
$$

If $f \in L^{p}\left(B_{2 R}\right)$ for $1<p<\infty$, then the solution $u \in W^{2, p}\left(B_{R}\right)$, and

$$
\left\|D^{2} u\right\|_{L^{p}\left(B_{R}\right)} \leq C_{p, n}\left(\|f\|_{L^{p}\left(B_{2 R}\right)}+\|u\|_{L^{1}\left(B_{2 R}\right)}\right)
$$

If $f \in L^{\infty}\left(B_{2 R}\right)$, then in general $u \notin W^{2, \infty}\left(B_{R}\right)$, but

$$
\left\|D^{2} u\right\|_{B M O\left(B_{R}\right)} \leq C_{\infty, n}\left(\|f\|_{L^{\infty}\left(B_{2 R}\right)}+\|u\|_{L^{1}\left(B_{2 R}\right)}\right),
$$

here $C_{p, n}, C_{\infty, n}$ are dimensional constants.

\subsection{The obstacle problem}

In this section we state the regularity of the solution to the following obstacle problem,

$$
\min (-\Delta u+f, u-\psi)=0 \text { in } \Omega
$$

with boundary conditions $u-g \in W_{0}^{1,2}(\Omega)$.

Here we will omit the variational formulation of the problem, the first regularity results and will state the $C^{1,1}$-regularity of the solutions referring to the book [8].

In order to be consistent with the assumptions in our paper, we will assume that $f \in C^{\alpha}$ and the obstacle $\psi \in C^{2, \alpha}$, although these assumptions can be weakened.

Theorem 3. Assume that $f \in C^{\alpha}$ and $\psi \in C^{2, \alpha}$, and $u$ solves the obstacle problem

$$
\min (-\Delta u+f, u-\psi)=0 \text { a.e. in } \Omega .
$$

Then $u \in C^{1,1}\left(\Omega^{\prime}\right)$ for every $\Omega^{\prime} \Subset \Omega$, and

$$
\|u\|_{C^{1,1}\left(\Omega^{\prime}\right)} \leq C\left(\|u\|_{L^{\infty}(\Omega)}+\|f\|_{C^{0, \alpha}(\Omega)}+\|\psi\|_{C^{2, \alpha}(\Omega)}\right),
$$

where the constant $C$ depends on the dimension and on the subset $\Omega^{\prime} \Subset \Omega$.

\section{Existence of $C^{1, \alpha}$ solutions}

We consider the system (1) with boundary conditions $u^{i}=g^{i}$ on $\partial \Omega, g^{i} \in C^{2}$. Then we also need to impose the following compatibility condition on the boundary data:

$$
g^{1}-g^{2}+\psi^{1} \geq 0, \text { and } g^{2}-g^{1}+\psi^{2} \geq 0 \text { on } \partial \Omega .
$$

Clearly, without the compatibility conditions, there are no solutions to (1) achieving the boundary data. 
We are interested in deriving $C^{1,1}$-regularity for the solutions to our system, which is the best regularity one can expect. Throughout our discussion we will assume that

$$
f^{1}, f^{2} \in C^{\alpha}(\Omega), \text { and } \psi^{1}, \psi^{2} \in C^{2, \alpha}(\Omega),
$$

for some $0<\alpha<1$. These are natural assumptions, since $f$ being bounded or continuous, is not enough for its Newtonian potential to be $C^{1,1}$. We also provide a one-dimensional counterexample to the existence of solutions in case the switching costs are not smooth.

Example 1 (Diogo Gomes). Consider the following system in the interval $(-1,1)$ with zero Dirichlet boundary conditions,

$$
\left\{\begin{array}{l}
\min \left(-\left(u_{1}\right)_{x x}, u_{1}-u_{2}+(1-|x|) \cos \left(\frac{\pi}{1-|x|}\right)\right)=0, \\
\min \left(-\left(u_{2}\right)_{x x}, u_{2}-u_{1}+(1-|x|)\left(1-\cos \left(\frac{\pi}{1-|x|}\right)\right)=0 .\right.
\end{array}\right.
$$

Then the value function of the corresponding optimal control problem is not finite.

Proof. In our example the running costs are identically zero, the switching costs satisfy the nonnegative loop assumption $\psi_{1}(x)+\psi_{2}(x)>0$ in $(-1,1)$, and the compatibility condition on the boundary $\psi^{1}( \pm 1)=\psi^{2}( \pm 1)=0$.

The example illustrates that when the switching costs are not smooth, then the negative values give infinity growth to the value function of the corresponding optimal control problem. In order to show this, we choose optimal controls $i(t)$ as follows: the switching occurs at times $t_{k}$ where $\frac{\pi}{1-\left|x\left(t_{k}\right)\right|}=\pi k$ : When $\frac{\pi}{1-\left|x\left(t_{k}\right)\right|}=\pi k=\pi(2 n+1), n \in \mathbb{N}_{0}$, we switch from regime 1 to regime 2 gaining $\frac{1}{2 n+1}$ and for the values $\frac{\pi}{1-\left|x\left(t_{k}\right)\right|}=\pi k=2 \pi n$ we switch back from regime 2 to 1 paying zero cost, and so

$$
u_{i}(x) \geq-\sum_{0 \leq t \leq T_{\partial \Omega}} \psi_{i\left(t_{k}\right), i\left(t_{k+1}\right)}(x(t))=\sum \frac{1}{2 n+1} .
$$

Then the conclusion follows from the divergence of harmonic series.

\subsection{Penalization method}

In this section we approximate the system (1) with a smooth penalized system. Let us take any smooth nonpositive function $\beta: \mathbb{R} \rightarrow(-\infty, 0]$, such that

$$
\begin{array}{r}
\beta(s)=0 \text { for } s \geq 0, \\
\beta(s)<0 \text { for } s<0 \text { and } \\
0<\beta^{\prime}(s) \leq 1 \text { for } s<0, \\
\lim _{s \rightarrow-\infty} \beta(s)=-\infty
\end{array}
$$

Next we consider the following penalization function $\beta_{\varepsilon}(s)=\beta(s / \varepsilon)$, for $s \in \mathbb{R}, \varepsilon>0$, and the corresponding penalized system

$$
\left\{\begin{array}{l}
-\Delta u_{\varepsilon}^{1}+f^{1}+\beta_{\varepsilon}\left(u_{\varepsilon}^{1}-u_{\varepsilon}^{2}+\psi^{1}\right)=0 \\
-\Delta u_{\varepsilon}^{2}+f^{2}+\beta_{\varepsilon}\left(u_{\varepsilon}^{2}-u_{\varepsilon}^{1}+\psi^{2}\right)=0
\end{array}\right.
$$

with boundary conditions $u_{\varepsilon}^{i}=g^{i}$ on $\partial \Omega$.

For $\varepsilon>0$ fixed, the penalized system (7) can be solved by several methods. In the paper [4] the authors use nonlinear functional analysis methods in order to derive the existence of classical solutions, that is $u_{\varepsilon}^{i} \in C^{2}(\Omega)$, assuming that the switching costs are positive constants. The proof is rather technical, however it works line for line in our case with variable switching costs, therefore we omit it.

Lemma 1. Under the assumptions (5) and (6) the solutions to the penalized system (7), $u_{\varepsilon}^{i}$ satisfy the following estimates for every $\varepsilon>0$ 
i.)

$$
-\max _{i}\left\|f^{i}\right\|_{L^{\infty}} \leq-\Delta u_{\varepsilon}^{i} \leq \max _{i}\left\|\Delta \psi^{i}\right\|_{L^{\infty}}+3 \max _{i}\left\|f^{i}\right\|_{L^{\infty}}
$$

ii.)

$$
u_{\varepsilon}^{1}-u_{\varepsilon}^{2}+\psi^{1} \geq-C \varepsilon \text { and } u_{\varepsilon}^{2}-u_{\varepsilon}^{1}+\psi^{2} \geq-C \varepsilon
$$

In ii.) the constant $C>0$ depends only on the given data and can be computed explicitly in terms of $\beta$.

Proof. For our convenience, let us denote $\theta_{\varepsilon}^{1}=u_{\varepsilon}^{1}-u_{\varepsilon}^{2}+\psi^{1}$ and $\theta_{\varepsilon}^{2}=u_{\varepsilon}^{2}-u_{\varepsilon}^{1}+\psi^{2}$, and observe that $\theta_{\varepsilon}^{1}$ and $\theta_{\varepsilon}^{2}$ cannot be negative at the same time according to the nonnegative loop assumption.

Now let us fix $\varepsilon>0$, and consider the function $\beta_{\varepsilon}\left(\theta_{\varepsilon}^{i}(x)\right), x \in \Omega$. It is bounded from above by 0 , our aim is to prove that $\beta_{\varepsilon}\left(\theta_{\varepsilon}^{i}(x)\right)$ is bounded from below. Let $x_{0}=x_{0}(\varepsilon)$ be a point of minimum for the function $\beta_{\varepsilon}\left(\theta_{\varepsilon}^{1}(x)\right)$, moreover without loss of generality, we may assume that

$$
\min _{i=1,2 ; x \in \bar{\Omega}} \beta_{\varepsilon}\left(\theta_{\varepsilon}^{i}(x)\right)=\beta_{\varepsilon}\left(\theta_{\varepsilon}^{1}\left(x_{0}\right)\right)<0 .
$$

If $x_{0} \in \partial \Omega$, then $\beta_{\varepsilon}\left(\theta_{\varepsilon}^{1}\left(x_{0}\right)\right)=0$ according to (5). Therefore $x_{0} \in \Omega$ is an interior point, and $\beta_{\varepsilon}\left(\theta_{\varepsilon}^{1}\left(x_{0}\right)\right)<0$. Then $\theta_{\varepsilon}^{1}\left(x_{0}\right)<0$, and since $\theta_{\varepsilon}^{1}+\theta_{\varepsilon}^{2} \geq 0$, we get $\theta_{\varepsilon}^{2}\left(x_{0}\right) \geq 0$ consequently $\beta_{\varepsilon}\left(\theta_{\varepsilon}^{2}\left(x_{0}\right)\right)=0$. Since $\beta_{\varepsilon}$ is nondecreasing and $\beta_{\varepsilon}(t)<0$ if and only if $t<0$, we get that

$$
\min _{i=1,2 ; x \in \bar{\Omega}} \theta_{\varepsilon}^{i}(x)=\theta_{\varepsilon}^{1}\left(x_{0}\right) .
$$

This implies that $\theta_{\varepsilon}^{1}=u_{\varepsilon}^{1}-u_{\varepsilon}^{2}+\psi^{1}$ achieves its minimum at an interior point $x_{0}$, hence $\Delta u_{\varepsilon}^{1}-\Delta u_{\varepsilon}^{2}+\Delta \psi^{1} \geq 0$ at $x_{0}$. The last inequality together with $-\Delta u_{\varepsilon}^{2}\left(x_{0}\right)+f^{2}\left(x_{0}\right)=0$ shows that

$$
\begin{aligned}
\beta_{\varepsilon}\left(\theta_{\varepsilon}^{1}\left(x_{0}\right)\right)= & \Delta u_{\varepsilon}^{1}\left(x_{0}\right)-f^{1}\left(x_{0}\right)= \\
& \Delta u_{\varepsilon}^{1}\left(x_{0}\right)-\Delta u_{\varepsilon}^{2}\left(x_{0}\right)+f^{2}\left(x_{0}\right)-f^{1}\left(x_{0}\right) \geq-\Delta \psi^{1}\left(x_{0}\right)+f^{2}\left(x_{0}\right)-f^{1}\left(x_{0}\right) .
\end{aligned}
$$

The estimate above is true for any $\varepsilon>0$, and therefore it proves the right inequality in $i$.). The left inequality in $i$.) is a direct consequence of $-\beta_{\varepsilon} \geq 0$.

In order to prove $i i$.), we recall that $\lim _{s \rightarrow-\infty} \beta(s)=-\infty$, and $\beta_{\varepsilon}(s)=\beta(s / \varepsilon)$, hence $\beta_{\varepsilon}\left(\theta_{\varepsilon}^{i}\right)$ is bounded implies that $\frac{\theta_{\varepsilon}^{i}}{\varepsilon}$ is uniformly bounded from below by a negative constant $-C \leq 0$. This finishes the proof of point ii.) in our lemma.

Using the Sobolev embedding theorem and Calderon-Zygmund estimates, we can conclude that the functions $u_{\varepsilon}^{i}$ are uniformly bounded in $W^{2, p}$ for every $1<p<\infty$. Therefore through a subsequence $u_{\varepsilon}^{i}$ converges to a function $u_{0}^{i}$ locally weakly in $W^{2, p}$ and strongly in $C^{1, \gamma}$ for every $0<\gamma<1$.

Now we proceed to prove the existence of solutions to system (1).

Proposition 1. Let $\left(u_{0}^{1}, u_{0}^{2}\right)=\lim _{\varepsilon \rightarrow 0}\left(u_{\varepsilon}^{1}, u_{\varepsilon}^{2}\right)$ through a subsequence weakly in $W^{2, p}$ and strongly in $C^{1, \gamma}$. Then $\left(u_{0}^{1}, u_{0}^{2}\right)$ solves the following system

$$
\left\{\begin{array}{l}
\min \left(-\Delta u_{0}^{1}+f^{1}, u_{0}^{1}-u_{0}^{2}+\psi^{1}\right)=0, \\
\min \left(-\Delta u_{0}^{2}+f^{2}, u_{0}^{2}-u_{0}^{1}+\psi^{2}\right)=0 \\
\min \left(-\Delta u_{0}^{1}+f^{1},-\Delta u_{0}^{2}+f^{2}\right)=0
\end{array}\right.
$$

in a strong sense, i.e. $u_{0}^{i}-u_{0}^{j}+\psi^{i} \geq 0$ and if we have a strict inequality at some point then $u_{0}^{i}$ satisfies $\Delta u_{0}^{i}=f^{i}$ in a neighborhood of that point, and $-\Delta u_{0}^{i}+f^{i} \geq 0$ a.e.

Proof. The property ii.) in Lemma 1, together with the strong convergence in $C^{1, \gamma}$ shows that $u_{0}^{1}-u_{0}^{2}+\psi^{1} \geq 0$ and $u_{0}^{2}-u_{0}^{1}+\psi^{2} \geq 0$. If $u_{0}^{1}\left(x_{0}\right)-u_{0}^{2}\left(x_{0}\right)+\psi^{1}\left(x_{0}\right)>0$, then the strict inequality $u_{\varepsilon}^{1}-u_{\varepsilon}^{2}+\psi^{1}>0$ holds in a small 
ball $B_{r}\left(x_{0}\right)$, centered at $x_{0}$ for $\varepsilon>0$ small enough. Then it follows that $-\Delta u_{\varepsilon}^{1}+f^{1}=0$ in $B_{r}\left(x_{0}\right)$, and we know that $\left\|\Delta u_{\varepsilon}^{1}\right\|_{L^{\infty}}$ is uniformly bounded, therefore through a subsequence, $\Delta u_{\varepsilon}^{1} \rightarrow \Delta u_{0}^{1}$ a.e. as $\varepsilon \rightarrow 0$, consequently $-\Delta u_{0}^{1}+f^{1}=0$ a.e. in $B_{r}\left(x_{0}\right)$. Moreover, since $f^{1} \in C^{\alpha}$, we know that $u_{0}^{1}$ is a classical solution to $-\Delta u_{0}^{1}+f^{1}=0$ in the ball $B_{r}\left(x_{0}\right)$.

The solutions of the penalized system satisfy the equation

$$
\min \left(-\Delta u_{\varepsilon}^{1}+f^{1},-\Delta u_{\varepsilon}^{2}+f^{2}\right)=0 .
$$

After passing to a limit through a subsequence, we get the following

$$
\min \left(-\Delta u_{0}^{1}+f^{1},-\Delta u_{0}^{2}+f^{2}\right)=0 \text { a.e. }
$$

Proposition 1 shows that there exists $\left(u_{0}^{1}, u_{0}^{2}\right), u_{0}^{i} \in W^{2, p}, \forall p<\infty$ solving (1) in a strong sense. According to Lemma $1, u_{0}^{i}$ has the following property

$$
\left\|\Delta u_{0}^{i}\right\|_{L^{\infty}} \leq \max _{i}\left\|\Delta \psi^{i}\right\|_{L^{\infty}}+3 \max _{i}\left\|f^{i}\right\|_{L^{\infty}},
$$

which will be relevant for deriving further regularity of solutions.

Furthermore, Proposition 1 tells us that the solution we get via the penalization method, solves an extra equation, which turns out to be very important in the discussion of the uniqueness.

\subsection{Uniqueness}

It has been shown in the paper [7] that if there are no zero loops, then the solution to the system (1) is unique. Here we give a counterexample showing that the uniqueness does not hold in case there are zero loops.

Example 2 (Diogo Gomes). The following system

$$
\left\{\begin{array}{l}
\min \left(-\Delta u^{1}-M, u^{1}-u^{2}+\psi\right)=0 \\
\min \left(-\Delta u^{2}+M, u^{2}-u^{1}-\psi\right)=0,
\end{array}\right.
$$

with given boundary conditions $u^{i}=g^{i}, g^{1}-g^{2}+\psi=0$ on $\partial \Omega$, admits infinitely many solutions, provided $2 M>$ $\|\Delta \psi\|_{L^{\infty}}$.

Moreover, (10) admits solutions $u^{1}, u^{2} \notin C^{1,1}$.

Proof. Let $\left(u^{1}, u^{2}\right)$ be a solution to the system (10). Since both $u^{1}-u^{2}+\psi \geq 0$ and $u^{2}-u^{1}-\psi \geq 0$, it follows that $u^{1}-u^{2}+\psi \equiv 0$, therefore $-\Delta u^{1}=-\Delta u^{2}+\Delta \psi$.

Now let us take any $u^{1} \in W^{2, p}, p>n, u^{1}=g^{1}$ on $\partial \Omega$, such that $-\Delta u^{1}-M \geq 0$ a.e. Then the function $u^{2}=u^{1}+\psi$ satisfies the boundary conditions $u^{2}=g^{2}$ on $\partial \Omega$, and $-\Delta u^{2}+M \geq 0$ a.e. since $2 M>\|\Delta \psi\|_{L^{\infty}}$. Thus we get infinitely many solutions of the form $\left(u^{1}, u^{1}+\psi\right)$, which may not be $\bar{C}^{1,1}$.

We observe that if the zero loop set is empty, then the equation $\min \left(-\Delta u^{1}+f^{1},-\Delta u^{2}+f^{2}\right)=0$ is satisfied automatically. Under the nonnegative loop assumption, we saw that there exists a solution to system (1) also solving system (8). Next we show that the system (8) has a unique solution, which is actually the minimal solution to (1).

Proposition 2. The system (8) has a unique solution $\left(u_{0}^{1}, u_{0}^{2}\right)$ in $W^{2, p}$ for every $p<\infty$.

Proof. Let us assume that $\left(u^{1}, u^{2}\right)$ is a solution to system (8), then the difference $U=u^{1}-u^{2}$ solves the following double-obstacle problem in $\Omega$ :

$$
\left\{\begin{array}{l}
-\Delta U+f^{1}-f^{2} \leq 0 \text { a.e. if } U>-\psi^{1} \\
-\Delta U+f^{1}-f^{2} \geq 0 \text { a.e. if } U<\psi^{2} \\
-\psi^{1} \leq U \leq \psi^{2}
\end{array}\right.
$$

with boundary conditions $U=g^{1}-g^{2}$ on $\partial \Omega$. 
It is well-known that the solution to the double-obstacle problem with given boundary data is unique in $W^{2, p}$. Indeed, let $V$ be another solution, then without loss of generality, we may assume that $\max _{x \in \Omega}(U-V)=U\left(x_{0}\right)-$ $V\left(x_{0}\right)>0$. Then in a small ball $B_{r}\left(x_{0}\right)$, one has $U-V>0$, and $U-V$ has a maximum at $x_{0}$. The inequality $U>V \geq-\psi^{1}$ implies that $U>-\psi^{1}$ in $B_{r}\left(x_{0}\right)$, hence $-\Delta U-f^{1}+f^{2} \leq 0$. Similarly, $V<\psi^{2}$ in $B_{r}\left(x_{0}\right)$ and therefore $-\Delta V-f^{1}+f^{2} \geq 0$. After combining the inequalities $-\Delta U-f^{1}+f^{2} \leq 0$ and $-\Delta V-f^{1}+f^{2} \geq 0$, we see that $U-V$ is a subharmonic function in the ball $B_{r}\left(x_{0}\right)$. Recalling that $U-V$ has a maximum at an interior point $x_{0}$, we get a contradiction to the maximum principle for subharmonic functions.

Now let us assume that $\left(v^{1}, v^{2}\right)$ is another solution to system (8), then $u^{1}-u^{2} \equiv v^{1}-v^{2}$ in $\Omega$. Denote $h=$ $u^{1}-v^{1} \equiv u^{2}-v^{2}$ in $\Omega$, then $h=0$ on $\partial \Omega$.

Now let us plugg-in $v^{1}=u^{1}-h$ and $v^{2}=u^{2}-h$ to the equation

$$
\begin{aligned}
0= & \min \left(-\Delta v^{1}+f^{1},-\Delta v^{2}+f^{2}\right)= \\
& \min \left(-\Delta u^{1}+f^{1},-\Delta u^{2}+f^{2}\right)+\Delta h=\Delta h \text { a.e. }
\end{aligned}
$$

Then it follows that $\Delta h=0$ a.e. in $\Omega, h \in W^{2, p}(\Omega)$, for every $1<p<\infty$, hence $h$ is a harmonic function. Then the difference $u^{i}-v^{i}$ is a harmonic function in $\Omega$, vanishing on the boundary, therefore $u^{i}-v^{i} \equiv 0$, according to the maximum principle for harmonic functions.

Corollary 1. The solution to the system (8) is the minimal solution to system (1), that is if $\left(v^{1}, v^{2}\right)$ solves (1), then $u_{0}^{1} \leq v^{1}$ and $u_{0}^{2} \leq v^{2}$.

Proof. Assume $\left(v^{1}, v^{2}\right)$ solves (1) with given boundary conditions, and let $\omega=\min \left(-\Delta v^{1}+f^{1},-\Delta v^{2}+f^{2}\right)$, then $\omega \geq 0$ a.e., $\omega \in L^{\infty}$. Let $h$ be the solution to $\Delta h=\omega$ in $\Omega$ with zero Dirichlet boundary conditions on $\partial \Omega$. Then according to the weak maximum principle for subharmonic functions, we get that $h \leq 0$ in $\Omega$.

Now we note that the pair $\left(v^{1}+h, v^{2}+h\right)$ solves the system (8) with the same boundary conditions as $\left(u_{0}^{1}, u_{0}^{2}\right)$, hence $v^{1}+h=u_{0}^{1}$ and $v^{2}+h=u_{0}^{2}$. Then the minimality of $\left(u_{0}^{1}, u_{0}^{2}\right)$ follows from nonpositivity of $h$.

From now on we will be interested in studying the regularity for the minimal solutions. As Example 2 shows, there is no hope to get $C^{1,1}$-regularity for non-minimal solutions.

\section{Optimal regularity of the solutions}

In this section we prove that the solution to the system (8) is locally $C^{1,1}$, if $\mathscr{L}=\overline{\mathscr{L}^{0}}$. In particular we study the regularity of the solutions on $\partial \mathscr{L}$, the boundary of the zero-loop set.

Before proceeding to the discussion of $C^{1,1}$-regularity, let us rewrite our system in a more convenient way. We have assumed that $f^{1}, f^{2} \in C^{\alpha}$, therefore there exist $v^{1}, v^{2} \in C_{l o c}^{2, \alpha}$ solving the Poisson equation $\Delta v^{i}=f^{i}$ in $\Omega$. Recall that $\left(u_{0}^{1}, u_{0}^{2}\right)$ is the solution to system (8), and define $u^{i}=u_{0}^{i}-v^{i}$, then $u_{0}^{i}$ is as regular as $u^{i}$ up to $C^{2, \alpha}$, and $\left(u^{1}, u^{2}\right)$ solves the following system

$$
\left\{\begin{array}{l}
\min \left(-\Delta u^{1}, u^{1}-u^{2}+\varphi^{1}\right)=0, \\
\min \left(-\Delta u^{2}, u^{2}-u^{1}+\varphi^{2}\right)=0 \\
\min \left(-\Delta u^{1},-\Delta u^{2}\right)=0
\end{array}\right.
$$

Here $\varphi^{1}=v^{1}-v^{2}+\psi^{1}$ and $\varphi^{2}=v^{2}-v^{1}+\psi^{2}$ are the new switching cost functions preserving the loop condition $\varphi^{1}+\varphi^{2} \equiv \psi^{1}+\psi^{2}$, and $\varphi^{1}, \varphi^{2} \in C_{l o c}^{2, \alpha}$.

From now on we will be focused on studying the regularity of $\left(u^{1}, u^{2}\right)$ solving the system (11).

We define the open set $\Omega_{1}:=\cup B_{r}\left(x_{0}, u^{1}\right)$, where the union is taken over the balls $B_{r}\left(x_{0}, u^{1}\right)$, such that $-\Delta u^{1}>0$ a.e. in $B_{r}\left(x_{0}, u^{1}\right)$. Similarly we define the set $\Omega_{2}$ corresponding to the function $u^{2}$, and let $\Omega_{12}=\Omega \backslash \overline{\Omega_{1} \cup \Omega_{2}}$. Then $\Omega_{1}, \Omega_{2}$ and $\Omega_{12}$ are disjoint open sets, and since $\varphi^{1}, \varphi^{2} \in C^{2, \alpha}$,

$$
\begin{aligned}
&-\Delta u^{1}=\Delta \varphi^{1}>0,-\Delta u^{2}=0 \text { in } \Omega_{1}, \\
&-\Delta u^{2}=\Delta \varphi^{2}>0,-\Delta u^{1}=0 \text { in } \Omega_{2}, \\
&-\Delta u^{1}=0,-\Delta u^{2}=0 \text { in } \Omega_{12} .
\end{aligned}
$$


In the set $\Omega \backslash \overline{\Omega_{1}}$ we get $-\Delta u^{1}=0$, and the function $u^{2}$ solves the obstacle problem $\min \left(-\Delta u^{2}, u^{2}-u^{1}+\varphi^{2}\right)=0$, with a $C^{2, \alpha}$ obstacle $u^{1}-\varphi^{2}$. Therefore $u^{2}$ is locally $C^{1,1}$ in $\Omega \backslash \overline{\Omega_{1}}$. Similarly we get that $u^{2}$ is locally $C^{1,1}$ in $\Omega \backslash \overline{\Omega_{2}}$.

Next we need to study the regularity of the solution in a neighborhood of the set $\partial \Omega_{1} \cap \partial \Omega_{2} \cap \Omega$. Let us note that it is contained in the zero loop set, $\partial \Omega_{1} \cap \partial \Omega_{2} \subset \mathscr{L}$, since $u^{1}-u^{2}+\varphi^{1} \equiv 0$ in $\Omega_{1}$ and $u^{2}-u^{1}+\varphi^{2} \equiv 0$ in $\Omega_{2}$. In the interior of the zero loop set the system (11) reduces to the equation

$$
-\Delta u^{1}=\left(\Delta \varphi^{1}\right)^{+}, u^{2}=u^{1}+\varphi^{1} \text { in } \mathscr{L}^{0} .
$$

From the classical theory, solutions to the equation (12) are locally $C^{2, \alpha}$ if $\Delta \varphi^{1} \in C^{\alpha}$. So in a neighborhood of the points $x \in \partial \Omega_{1} \cap \partial \Omega_{2}$ and $x \in \mathscr{L}^{0}$, the solution is $C^{2, \alpha}$.

It remains to study the regularity of $\left(u^{1}, u^{2}\right)$ at the points $x_{0} \in \partial \Omega_{1} \cap \partial \Omega_{2} \cap \partial \mathscr{L}$, called a "meeting" point. In this section we show that $u^{1}$ and $u^{2}$ are actually $C^{2, \alpha}$-regular at such points.

For simplicity, let us study the system locally in the unit ball $B_{1}$, assuming that $0 \in \partial \mathscr{L} \cap \partial \Omega_{1} \cap \partial \Omega_{2}$. We can always come to such a situation with a change of variables.

\subsection{Blow-up procedure}

Assume that $\left(u^{1}, u^{2}\right)$ solves system (11) in the unit ball $B_{1}$, and $0 \in \partial \mathscr{L}=\partial \mathscr{L}^{0}$ is a meeting point, and let us study the regularity of the functions $u^{1}$ and $u^{2}$ at 0 .

Definition 3. For a function $u \in W^{2,2}$, define $\Pi(u(x), r)=p_{r}(x)$, where $p_{r}(x)=x \cdot A_{r} \cdot x+b_{r} \cdot x+c_{r}$ is a second order polynomial with the matrix $A_{r}$, vector $b_{r}$ and scalar $c_{r}$ minimizing the following expression

$$
\min _{A, b, c} \int_{B_{r}}\left(\left|D^{2} u-2 A_{r}\right|^{2}+\left|\nabla u-b_{r}\right|^{2}+\left|u-c_{r}\right|^{2}\right) d x .
$$

Then $\Pi(u(r x), 1)=p_{r}(r x)$, and it is easy to see that

$$
p_{r}(x)=\frac{1}{2} x \cdot\left(D^{2} u\right)_{r} \cdot x+(\nabla u)_{r} \cdot x+(u)_{r},
$$

where $(u)_{r}:=(u)_{r, 0}$, and $(u)_{r, x_{0}}$ is the average of $u$ over the ball $B_{r}\left(x_{0}\right)=\left\{x \in \mathbb{R}^{n}|| x-x_{0} \mid<r\right\}$,

$$
(u)_{r, x_{0}}=\frac{1}{\left|B_{r}\right|} \int_{B_{r}\left(x_{0}\right)} u \text {. }
$$

Let us recall that $\left(u^{1}, u^{2}\right) \in W^{2,2}$ is the solution to (11) in the unit ball $B_{1}$, and denote $A_{r}^{i}=\frac{1}{2}\left(D^{2} u^{i}\right)_{r}, b_{r}^{i}=\left(\nabla u^{i}\right)_{r}$, $c_{r}^{i}=\left(u^{i}\right)_{r}$, for $i \in\{1,2\}$, then $\Pi\left(u^{i}(r x), 1\right)=r^{2} x \cdot A_{r}^{i} \cdot x+r b_{r}^{i} \cdot x+c_{r}^{i}$.

Next for any $0<r<1$, we define

$$
v_{r}^{i}(x)=\frac{u^{i}(r x)-\Pi\left(u^{i}(r x), 1\right)}{S(r)},
$$

where $S(r)$ is chosen such that $\max _{i}\left\|D^{2} v_{r}^{i}\right\|_{L^{2}\left(B_{1}\right)}=1$. Our aim is to describe the rate of convergence of $S(r)$ as $r$ goes to zero.

It follows immediately from our definition of $S(r)$, and $B M O$-estimates that $\frac{S(r)}{r^{2}}$ is uniformly bounded from above. In order to show this, let us recall that $\left\|\Delta u^{i}\right\|_{L^{\infty}} \leq \max _{i}\left\|\Delta \varphi^{i}\right\|_{L^{\infty}}$, hence $D^{2} u^{i} \in B M O$ locally, with the following estimate

$$
\left\|D^{2} u^{i}\right\|_{B M O\left(B_{\frac{1}{2}}\right)} \leq C\left(\max _{i}\left\|\Delta \varphi^{i}\right\|_{L^{\infty}}+\left\|u^{i}\right\|_{L^{2}\left(B_{1}\right)}\right) .
$$

Without loss of generality, we may assume that $\left\|D^{2} v_{r}^{1}\right\|_{L^{2}\left(B_{1}\right)}=1$, for a fixed $r>0$, then

$$
\frac{S(r)}{r^{2}}=\left\|D^{2} u^{1}(r x)-2 A_{r}^{1}\right\|_{L^{2}\left(B_{1}\right)} .
$$


A change of variable will give us

$$
\begin{aligned}
\left(\frac{S(r)}{r^{2}}\right)^{2}= & \frac{1}{r^{n}} \int_{B_{r}}\left|D^{2} u^{1}(y)-2 A_{r}^{1}\right|^{2} d y= \\
& \frac{1}{r^{n}} \int_{B_{r}}\left|D^{2} u^{1}(y)-\left(D^{2} u^{1}\right)_{r}\right|^{2} d y \leq C\left(\max _{i}\left\|\Delta \varphi^{i}\right\|_{L^{\infty}}+\left\|u^{1}\right\|_{L^{2}\left(B_{1}\right)}\right)^{2},
\end{aligned}
$$

therefore $\forall r<\frac{1}{2}$

$$
\frac{S(r)}{r^{2}} \leq C\left(\max _{i}\left\|\Delta \varphi^{i}\right\|_{L^{\infty}}+\max _{i}\left\|u^{i}\right\|_{L^{2}\left(B_{1}\right)}\right):=C_{0} .
$$

So $S(r)$ has at least quadratic decay as $r \rightarrow 0$. Next we improve the estimate, showing that actually $S(r) \leq C_{0} r^{2+\alpha}$ for $r>0$ small enough.

Proposition 3. Let $\varphi^{1}, \varphi^{2} \in C^{2, \alpha}$ for some $0<\alpha<1$, and $\mathscr{L}=\overline{\mathscr{L}^{0}}$, then the function $\frac{S(r)}{r^{2+\alpha}}$ is uniformly bounded as $r$ goes to zero

$$
\frac{S(r)}{r^{2+\alpha}} \leq C\left(\max _{i}\left\|\Delta \varphi^{i}\right\|_{L^{\infty}}+\max _{i}\left\|u^{i}\right\|_{L^{2}\left(B_{1}\right)}\right),
$$

where $C$ is a dimensional constant.

Proof. Let us start with an important observation: The assumptions $0 \in \partial \mathscr{L} \cap \partial \Omega_{1} \cap \partial \Omega_{2}, \mathscr{L}=\left\{\varphi^{1}+\varphi^{2}=0\right\}$, $\mathscr{L}=\overline{\mathscr{L}^{0}}$ and $\varphi^{1}, \varphi^{2} \in C^{2, \alpha}$ imply that $\Delta \varphi^{1}(0)+\Delta \varphi^{2}(0)=0$. On the other hand $\Delta \varphi^{1}>0$ in $\Omega_{1}$ and $\Delta \varphi^{2}>0$ in $\Omega_{2}$, therefore $\Delta \varphi^{1}(0)=\Delta \varphi^{2}(0)=0$.

Next we show that $\varphi^{i} \in C^{2, \alpha}$ together with $\Delta \varphi^{i}(0)=0$, provide the growth estimate (14). The proof is based on an argument of contradiction, assume that $\frac{S(r)}{r^{2+\alpha}}$ is not bounded, then there exists a sequence $r_{k} \rightarrow 0$ as $k \rightarrow \infty$, such that $S\left(r_{k}\right)=k r_{k}^{2+\alpha}$ and $S(r) \leq k r^{2+\alpha}$ for all $r \geq r_{k}$. Our aim is to study the convergence of the sequence $v_{k}^{i}:=v_{r_{k}}^{i}$ as $k \rightarrow \infty$. For that we will need some basic properties of the functions $v_{r}^{i}$, where $0<r<1$.

According to the definition of $S(r),\left\|D^{2} v_{r}^{i}\right\|_{L^{2}\left(B_{1}\right)} \leq 1$ for $r<1$. Then applying Poincaré's inequality for the function $\nabla v_{r}^{i}$ in the unit ball $B_{1}$, we get $\left\|\nabla v_{r}^{i}\right\|_{L^{2}\left(B_{1}\right)} \leq C_{n}$ for every $0<r<1$, since $\left(\nabla v_{r}^{i}\right)_{1}=0$. Next we study the average of $v_{r}^{i}$ in the unit ball

$$
\begin{aligned}
\left(v_{r}^{i}\right)_{1} & =\frac{\left(u^{i}\right)_{r}-\frac{n}{2} r^{2} \cdot \operatorname{tr}\left(D^{2} u^{i}\right)_{r} f_{B_{1}} x_{1}^{2} d x-r f_{B_{1}} x \cdot\left(\nabla u^{i}\right)_{r} d x-\left(u^{i}\right)_{r}}{S(r)} \\
& =-\alpha_{n} \frac{r^{2}\left(\Delta u^{i}(r x)\right)_{1}}{S(r)},
\end{aligned}
$$

where $\alpha_{n}=\frac{n}{2} f_{B_{1}} x_{1}^{2} d x$ is a dimensional constant. Now let us recall that $\left(u^{1}, u^{2}\right)$ solves (11), and therefore

$$
0 \leq-\Delta u^{i}(r x) \leq \max \left(0, \Delta \varphi^{i}(r x)\right) \leq\left\|\varphi^{i}\right\|_{C^{2, \alpha}} r^{\alpha}|x|^{\alpha},
$$

since $\Delta \varphi^{i}(0)=0, \varphi^{i} \in C^{2, \alpha}$. Hence we get $0 \leq\left(v_{r}^{i}\right)_{1} \leq C_{n} \frac{r^{2+\alpha}}{S(r)}\left\|\varphi^{i}\right\|_{C^{2, \alpha}}$, where $C_{n}>0$ is a dimensional constant. Next we apply Poincaré's inequality one more time, $\left\|v_{r}^{i}-\left(v_{r}^{i}\right)_{1}\right\|_{L^{2}\left(B_{1}\right)} \leq C_{n}\left\|\nabla v_{r}^{i}\right\|_{L^{2}\left(B_{1}\right)}$. Therefore we may conclude that

$$
\left\|\nabla v_{r}^{i}\right\|_{L^{2}\left(B_{1}\right)} \leq C,\left\|v_{r}^{i}\right\|_{L^{2}\left(B_{1}\right)} \leq C\left(1+\|\varphi\|_{C^{2, \alpha}} \frac{r^{2+\alpha}}{S(r)}\right),
$$

for every $0<r<1$, where the constant $C>0$ depends only on the dimension. 
Next by using (15) we want to estimate the $\left\|v_{k}^{i}\right\|_{W^{2,2}\left(B_{R}\right)}$ for $R<1 / r_{k}$ as $k \rightarrow \infty$. Let us start by looking at the expressions $\left|A_{2^{l} r_{k}}^{i}-A_{2^{l-1} r_{k}}^{i}\right|$, where $l \in \mathbb{N}$ and $r_{k}<1$ are chosen such that $s=r_{k} 2^{l-1} \leq \frac{1}{4}$. It follows from Minkowski's inequality, that

$$
\begin{aligned}
\left|A_{2 s}^{i}-A_{s}^{i}\right| & \leq\left(f_{B_{1}}\left|D^{2} u^{i}(x s)-2 A_{s}^{i}\right|^{2}\right)^{\frac{1}{2}}+\left(f_{B_{1}}\left|D^{2} u^{i}(x s)-2 A_{2 s}^{i}\right|^{2}\right)^{\frac{1}{2}} \\
& \leq \frac{S(s)}{s^{2}}+2^{\frac{n}{2}}\left(f_{B_{\frac{1}{2}}}\left|D^{2} u^{i}(2 x s)-2 A_{2 s}^{i}\right|^{2}\right)^{\frac{1}{2}} \leq \frac{S(s)}{s^{2}}+2^{\frac{n}{2}} \frac{S(2 s)}{4 s^{2}} .
\end{aligned}
$$

Hence

$$
\left|A_{2^{l} r_{k}}^{i}-A_{2^{l-1} r_{k}}^{i}\right| \leq k\left(1+2^{\frac{n}{2}+\alpha}\right)\left(r_{k} 2^{l-1}\right)^{\alpha},
$$

provided $r_{k} 2^{l-1} \leq \frac{1}{4}$.

Now let us take any $m \in \mathbb{N}$ such that $2^{m+1} r_{k} \leq 1$, then

$$
\begin{aligned}
\left(\int_{B_{2^{m}}}\left|D^{2} v_{k}^{i}(x)\right|^{2} d x\right)^{\frac{1}{2}} & =\frac{r_{k}^{2}}{S\left(r_{k}\right)}\left(\int_{B_{2^{m}}}\left|D^{2} u^{i}\left(r_{k} x\right)-2 A_{r_{k}}^{i}\right|^{2} d x\right)^{\frac{1}{2}} \\
& \leq \frac{2^{\frac{m n}{2}}}{k r_{k}^{\alpha}}\left(\int_{B_{1}}\left|D^{2} u^{i}\left(2^{m} r_{k} x\right)-2 A_{r_{k}}^{i}\right|^{2} d x\right)^{\frac{1}{2}} \\
& \leq \frac{2^{\frac{m n}{2}}}{k r_{k}^{\alpha}}\left(\left(\int_{B_{1}}\left|D^{2} u^{i}\left(2^{m} r_{k} x\right)-2 A_{2^{m} r_{k}}^{i}\right|^{2} d x\right)^{\frac{1}{2}}+\left|A_{2^{m} r_{k}}^{i}-A_{r_{k}}^{i}\right|\right) \\
& \leq \frac{2^{\frac{m n}{2}}}{k r_{k}^{\alpha}}\left(\frac{S\left(2^{m} r_{k}\right)}{\left(2^{m} r_{k}\right)^{2}}+\sum_{j=1}^{m}\left|A_{2^{j} r_{k}}^{i}-A_{2^{j-1} r_{k}}^{i}\right|\right) \\
& \leq \frac{2^{\frac{m n}{2}}}{k r_{k}^{\alpha}}\left(k 2^{m \alpha} r_{k}^{\alpha}+k 2^{n} r_{k}^{\alpha} \sum_{j=1}^{m} 2^{\alpha(j-1)}\right) \leq 2^{n+1} 2^{m\left(\frac{n}{2}+\alpha\right)} .
\end{aligned}
$$

For every $R<\frac{1}{2 r_{k}}$ we can find an $m \in \mathbb{N}$ such that $2^{m-1} \leq R<2^{m}$, and then applying the estimates above, we get

$$
\int_{B_{R}}\left|D^{2} v_{k}^{i}(x)\right|^{2} d x \leq C_{n} R^{n+2 \alpha},
$$

for every $R<\frac{1}{2 r_{k}}$, where $C_{n}$ is a dimensional constant. Then we can also show that $\left\|\nabla v_{r_{k}}^{i}\right\|_{L^{2}\left(B_{R}\right)}$ and $\left\|v_{r_{k}}^{i}\right\|_{L^{2}\left(B_{R}\right)}$ are bounded by a constant depending only on $R$. Indeed, applying the corresponding estimates for $A_{r_{k}}^{i}$, and the first inequality in (15), we get

$$
\left|b_{2^{l} r_{k}}^{i}-b_{2^{l-1} r_{k}}^{i}\right| \leq C_{n, \alpha} k\left(r_{k} 2^{l}\right)^{1+\alpha},
$$

and therefore

$$
\left|b_{R r_{k}}^{i}-b_{r_{k}}^{i}\right| \leq C_{n, \alpha} k\left(r_{k} R\right)^{1+\alpha} .
$$


Then Poincaré's inequality in a ball $B_{R}$ implies that

$$
\left\|\nabla v_{k}^{i}-\left(\nabla v_{k}^{i}\right)_{R}\right\|_{L^{2}\left(B_{R}\right)} \leq C_{n} R\left\|D^{2} v_{k}^{i}\right\|_{L^{2}\left(B_{R}\right)},
$$

and

$$
\left\|v_{k}^{i}-\left(v_{k}^{i}\right)_{R}\right\|_{L^{2}\left(B_{R}\right)} \leq C_{n} R\left\|\nabla v_{k}^{i}\right\|_{L^{2}\left(B_{R}\right)},
$$

where

$$
\begin{aligned}
\left(\nabla v_{k}^{i}\right)_{R} & =\frac{r_{k}}{S\left(r_{k}\right)}\left(\nabla u_{r_{k}}^{i}-r_{k} A_{r_{k}}^{i} \cdot x-b_{r_{k}}^{i}\right)_{R} \\
& =\frac{r_{k}}{S\left(r_{k}\right)}\left(\left(\nabla u^{i}\right)_{R r_{k}}-b_{r_{k}}^{i}\right)=\frac{r_{k}}{k r_{k}^{2+\alpha}}\left(b_{R r_{k}}^{i}-b_{r_{k}}^{i}\right),
\end{aligned}
$$

and

$$
\left(v_{k}^{i}\right)_{R}=\frac{1}{S\left(r_{k}\right)}\left(c_{R r_{k}}^{i}-c_{r_{k}}^{i}+\frac{n}{2} r_{k}^{2}\left(\Delta u^{i}\right)_{r_{k}}\left(x_{1}^{2}\right)_{R}\right) .
$$

Next let us observe that the second inequality in (15), with the corresponding estimates for $A_{r}^{i}$ and $b_{r}^{i}$ imply that

$$
\left|c_{R r_{k}}^{i}-c_{r_{k}}^{i}\right| \leq C k\left(R r_{k}\right)^{2+\alpha} .
$$

Then it follows from the triangle's inequality that

$$
\left\|\nabla v_{k}^{i}\right\|_{L^{2}\left(B_{R}\right)} \leq C\left(R^{\frac{n}{2}+1+\alpha}+R^{\frac{n}{2}} \frac{r_{k}}{k r_{k}^{2+\alpha}}\left|b_{R r_{k}}^{i}-b_{r_{k}}^{i}\right|\right) \leq C_{n} R^{\frac{n}{2}+1+\alpha},
$$

and also

$$
\begin{aligned}
\left\|v_{k}^{i}\right\|_{L^{2}\left(B_{R}\right) \leq} & C\left(R\left\|\nabla v_{k}^{i}\right\|_{L^{2}\left(B_{R}\right)}+R^{\frac{n}{2}}\left(v_{k}^{i}\right)_{R}\right) \leq \\
& C\left(R^{\frac{n}{2}+2+\alpha}+R^{\frac{n}{2}} \frac{\left|c_{R r_{k}}^{i}-c_{r_{k}}^{i}\right|}{S\left(r_{k}\right)}+\|\varphi\|_{C^{2, \alpha}} R^{\frac{n}{2}+2} \frac{r_{k}^{2+\alpha}}{S\left(r_{k}\right)}\right) \leq C^{\prime} R^{\frac{n}{2}+2+\alpha} .
\end{aligned}
$$

Therefore we have shown that the sequence $v_{k}^{i}$ is locally uniformly bounded in $W^{2,2}$, hence through a subsequence, $v_{k}^{i}$ converges weakly in $W^{2,2}\left(B_{R}\right)$, and strongly in $W^{1,2}\left(B_{R}\right)$, denote $v_{0}^{i}=\lim _{k \rightarrow \infty} v_{k}^{i}$ for $i=1$, 2 . Then the weak convergence of the second order derivatives implies that

$$
\int_{B_{R}}\left|D^{2} v_{0}^{i}(x)\right|^{2} d x \leq \limsup _{k \rightarrow \infty} \int_{B_{R}}\left|D^{2} v_{k}^{i}(x)\right|^{2} d x,
$$

and therefore

$$
\int_{B_{R}}\left|D^{2} v_{0}^{i}(x)\right|^{2} d x \leq C_{n} R^{n+2 \alpha} .
$$

Next we describe further properties of the limit functions, $v_{0}^{1}$ and $v_{0}^{2}$. Recall that

$$
v_{k}^{i}(x)=\frac{u^{i}\left(r_{k} x\right)-\Pi\left(u^{i}\left(r_{k} x\right), 1\right)}{S\left(r_{k}\right)},
$$

then we have

$$
-\Delta v_{k}^{i}(x)=\frac{r_{k}^{2}}{S\left(r_{k}\right)}\left(-\Delta u^{i}\left(r_{k} x\right)+\operatorname{tr} A_{r_{k}}^{i}\right) .
$$


Let us denote

$$
\begin{array}{r}
q_{k}^{1}(x)=\frac{p_{r_{k}}^{1}\left(r_{k} x\right)-p_{r_{k}}^{2}\left(r_{k} x\right)+\varphi^{1}\left(r_{k} x\right)}{S\left(r_{k}\right)}, \text { and } \\
q_{k}^{2}(x)=\frac{p_{r_{k}}^{2}\left(r_{k} x\right)-p_{r_{k}}^{1}\left(r_{k} x\right)+\varphi^{2}\left(r_{k} x\right)}{S\left(r_{k}\right)} .
\end{array}
$$

Then $\left(v_{k}^{1}, v_{k}^{2}\right)$ is a strong solution to the following system

$$
\left\{\begin{array}{l}
\min \left(-\Delta v_{k}^{1}-\frac{t r A_{r_{k}}^{1}}{k r_{k}^{\alpha}}, v_{k}^{1}-v_{k}^{2}+q_{k}^{1}\right)=0 \\
\min \left(-\Delta v_{k}^{2}-\frac{t r A_{r_{k}}^{2}}{k r_{k}^{\alpha}}, v_{k}^{2}-v_{k}^{1}+q_{k}^{2}\right)=0 \\
\min \left(-\Delta v_{k}^{1}-\frac{t r A_{r_{k}}^{1}}{k r_{k}^{\alpha}},-\Delta v_{k}^{2}-\frac{t r A_{r_{k}}^{2}}{k r_{k}^{\alpha}}\right)=0,
\end{array}\right.
$$

therefore

$$
-\Delta v_{k}^{1}(x)=\left\{\begin{array}{l}
\frac{t r A_{r_{k}}^{1}}{k r_{k}^{\alpha}}+\frac{\Delta \varphi^{1}\left(r_{k} x\right)}{k r_{k}^{\alpha}}, \text { if } r_{k} x \in \Omega_{1} \\
\frac{\operatorname{tr} A_{r_{k}}^{1}}{k r_{k}^{\alpha}}, \text { otherwise, }
\end{array}\right.
$$

and

$$
-\Delta v_{k}^{2}(x)=\left\{\begin{array}{l}
\frac{t r A_{r_{k}}^{2}}{k r_{k}^{\alpha}}+\frac{\Delta \varphi^{2}\left(r_{k} x\right)}{k r_{k}^{\alpha}}, \text { if } r_{k} x \in \Omega_{2} \\
\frac{\operatorname{tr} A_{r_{k}}^{2}}{k r_{k}^{\alpha}}, \text { otherwise. }
\end{array}\right.
$$

Then $\Delta \varphi^{i}(0)=0$, for $i=1,2$ together with $\varphi^{i} \in C^{2, \alpha}$, implies that

$$
\frac{\left\|\Delta \varphi^{i}\left(r_{k} \cdot\right)\right\|_{L^{2}\left(B_{R}\right)}}{k r_{k}^{\alpha}} \leq \frac{C_{n}}{k} R^{\frac{n}{2}+\alpha}\left\|\varphi^{i}\right\|_{C^{2, \alpha}} \rightarrow 0, \text { as } k \rightarrow \infty,
$$

for $i=1,2$, and for any fixed $1 \leq R<\infty$.

We have that $v_{k}^{i} \rightarrow v_{0}^{i}$ weakly in $W^{2,2}\left(B_{R}\right)$ and $v_{k}^{i} \rightarrow v_{0}^{i}$ in $W^{1,2}\left(B_{R}\right)$, therefore $\Delta v_{k}^{i} \rightarrow \Delta v_{0}^{i}$ weakly in $L^{2}\left(B_{R}\right)$, but $\Delta v_{k}^{i}=\frac{t r A_{r_{k}}^{i}}{k r_{k}^{\alpha}}+\frac{\Delta \varphi^{i}\left(r_{k} x\right)}{k r_{k}^{\alpha}} \chi_{\Omega_{i}}\left(r_{k} x\right)$, and $\left\|\frac{\Delta \varphi^{i}\left(r_{k} x\right)}{k r_{k}^{\alpha}} \chi_{\Omega_{i}}\left(r_{k} x\right)\right\|_{L^{2}\left(B_{R}\right)} \rightarrow 0$. Thus we may conclude that the sequence of numbers $\frac{\operatorname{tr} A_{r_{k}}^{i}}{k r_{k}^{\alpha}}$ converges, and denote $a^{i}:=\lim _{k \rightarrow \infty} \frac{\operatorname{tr} A_{r_{k}}^{i}}{k r_{k}^{\alpha}}$. Then $\left\|\Delta v_{k}^{i}-a^{i}\right\|_{L^{2}\left(B_{R}\right)} \rightarrow 0$ as $k \rightarrow \infty$ for every $1 \leq R<$ $\infty$. Therefore both $-\Delta v_{0}^{1}-a^{1} \equiv 0$ and $-\Delta v_{0}^{2}-a^{2} \equiv 0$ in $\mathbb{R}^{n}$.

We have shown that $v_{0}^{i}(x)-a^{i} \frac{|x|^{2}}{2 n}$ is a harmonic functions in $\mathbb{R}^{n}$. Hence the matrix $D^{2} v_{0}^{i}$ has harmonic entries $D^{k} v_{0}^{i}$, where $k$ is a multiindex, $|k|=2$. Next we can apply the estimates of the derivatives for harmonic functions and inequality (16), to get

$$
\begin{aligned}
\left|\nabla D^{k} v_{0}^{i}\left(x_{0}\right)\right| & \leq R^{-\frac{n}{2}-1}\left\|D^{k} v_{0}^{i}\right\|_{L^{2}\left(B_{R}\left(x_{0}\right)\right)} \\
& \leq R^{-\frac{n}{2}-1}\left\|D^{k} v_{0}^{i}\right\|_{L^{2}\left(B_{2 R}\right)} \leq C^{\prime} R^{-1+\alpha},
\end{aligned}
$$

provided $R>\left|x_{0}\right|$. Letting $R \rightarrow \infty$, we see that the derivatives of $D^{k} v_{0}^{i}$ are vanishing, hence $D^{2} v_{0}^{i}$ is a constant matrix, and therefore $v_{0}^{i}, i \in\{1,2\}$ is a second order polynomial.

According to our construction, $v_{0}^{i}$ are orthogonal to the second order polynomials in $L^{2}\left(B_{1}\right)$-sense, hence both $v_{0}^{1}$ and $v_{0}^{2}$ must be identically zero. Then the constants $a^{1}=a^{2}=0$, and $\left\|\Delta v_{k}^{i}\right\|_{L^{2}\left(B_{1}\right)} \rightarrow 0$ as $k \rightarrow \infty$, the latter contradicts to the condition $\max _{i}\left\|D^{2} v_{k}^{i}\right\|_{L^{2}\left(B_{1}\right)}=1$. 


\section{2. $C^{2, \alpha}$-regularity at the meeting points}

We start by showing that the approximating polynomials $p_{r}^{i}$ converge to a polynomial $p_{0}^{i}$, and describe the rate of convergence.

Lemma 2. Let $\left(u^{1}, u^{2}\right)$ be a solution to (11), and assume that $\varphi^{i} \in C^{2, \alpha}$. Let the polynomials $p_{r}^{i}$ be as in Definition 3, then there exists a polynomial $p_{0}^{i}$ such that

$$
\sup _{x \in B_{r}}\left|p_{r}^{i}(x)-p_{0}^{i}(x)\right| \leq C r^{2+\alpha} .
$$

Proof. The condition $\left\|D^{2} v_{r}^{i}\right\|_{L^{2}\left(B_{1}\right)} \leq 1$ with the inequality (14) implies that

$$
\left(\int_{B_{1}}\left|D^{2} u^{i}(r x)-D^{2} p_{r}^{i}\right|^{2} d x\right)^{\frac{1}{2}} \leq \frac{S(r)}{r^{2}} \leq C_{0} r^{\alpha}
$$

Recall that $A_{r}^{i}=D^{2} p_{r}^{i}$, then using the triangle inequality, and that $A_{r}^{i}$ is minimizing $\left\|D^{2} u^{i}(r x)-A\right\|_{L^{2}\left(B_{1}\right)}$ over matrices $A \in \mathbb{R}^{n} \times \mathbb{R}^{n}$, we get $\left|A_{r}^{i}-A_{\frac{r}{2}}^{i}\right| \leq C_{0} r^{\alpha}$ for all $0<r<1$. By taking $r=2^{-n}$, we see that $A_{2^{-n}}^{i}$ is a Cauchy sequence;

$$
\begin{aligned}
\left|A_{2^{-n}}^{i}-A_{2^{-n-m}}^{i}\right| \leq & \Sigma_{k=0}^{m-1}\left|A_{2^{-n-k}}^{i}-A_{2^{-n-k-1}}^{i}\right| \leq \\
& \Sigma_{k=0}^{m-1}\left(2^{-\alpha}\right)^{n+k}=2^{-\alpha n} \Sigma_{k=0}^{m-1} 2^{-\alpha k},
\end{aligned}
$$

from the convergence of the series $\Sigma\left(2^{-\alpha}\right)^{n}$, it follows that $A_{2^{-n}}^{i}$ converges to some matrix $A_{0}^{i}$ as $n$ goes to infinity. The inequality also provides the rate of convergence; for a fixed $n$, by letting $m$ go to infinity, we see that $\left|A_{2^{-n}}^{i}-A_{0}^{i}\right| \leq$ $C_{0} 2^{-n \alpha}$.

Moreover, we get the estimate

$$
\left|A_{r}^{i}-A_{0}^{i}\right| \leq C r^{\alpha},
$$

for $0<r<1$, by choosing $n$ so that $2^{-n-1}<r \leq 2^{-n}$.

Next we proceed to describe the rate of convergence of $b_{r}^{i}$ and $c_{r}^{i}$. We know that $b_{r}^{i}=\left(\nabla u^{i}\right)_{r}$, and $c_{r}^{i}=\left(u^{i}\right)_{r}$, taking into account that $u^{i} \in C^{1, \gamma}$, we see that $b_{r}^{i} \rightarrow \nabla u^{i}(0)$ and $c_{r}^{i} \rightarrow u(0)$ as $r \rightarrow 0$. Our aim is to show that actually

$$
\left|b_{r}^{i}-\nabla u^{i}(0)\right| \leq C r^{1+\alpha} \text { and }\left|c_{r}^{i}-u^{i}(0)\right| \leq C r^{2+\alpha} .
$$

Let us recall that $\left(\nabla v_{r}^{i}\right)_{1}=0$, and therefore Poincaré's inequality implies that

$$
\left\|\nabla v_{r}^{i}\right\|_{L^{2}\left(B_{1}\right)} \leq C\left\|D^{2} v_{r}^{i}\right\|_{L^{2}\left(B_{1}\right)} \leq C^{\prime} .
$$

Hence

$$
\left(\int_{B_{1}}\left|\nabla u^{i}(r x)-\nabla p_{r}^{i}(r x)\right|^{2} d x\right)^{\frac{1}{2}} \leq C^{\prime} \frac{S(r)}{r} \leq C r^{1+\alpha} .
$$

Taking into account that $b_{r}^{i}$ is minimizing $\left\|\nabla u^{i}(r x)-r x \cdot A_{r}^{i}-b\right\|_{L^{2}\left(B_{1}\right)}$ over $b \in \mathbb{R}^{n}$, and applying triangle's inequality we get $\left|b_{r}^{i}-\nabla u^{i}(0)\right| \leq C r^{1+\alpha}$.

Furthermore, using Poincaré's inequality once again, we see that

$$
\left(\int_{B_{1}}\left|u^{i}(r x)-p_{r}^{i}(r x)+r^{2}\left(\Delta u^{i}\right)_{r}\right|^{2} d x\right)^{\frac{1}{2}} \leq C^{\prime} S(r) \leq C r^{2+\alpha},
$$


then

$$
\left|c_{r}^{i}-u^{i}(0)\right| \leq C r^{2+\alpha},
$$

by using that $c_{r}^{i}$ is minimizing $\left\|u^{i}(r x)+r^{2}\left(\Delta u^{i}\right)_{r}-\frac{1}{2} r^{2} x \cdot A_{r}^{i} \cdot x-r b_{r}^{i} \cdot x-c\right\|_{L^{2}\left(B_{1}\right)}$ over $c \in \mathbb{R}$.

Finally, after combining our estimates for $A_{r}^{i}, b_{r}^{i}$ and $c_{r}^{i}$, we get (17), where

$$
p_{0}^{i}(x)=\frac{1}{2} x \cdot A_{0}^{i} \cdot x+\nabla u^{i}(0) \cdot x+u^{i}(0),
$$

for $i=1,2$.

Corollary 2. Under the assumptions of Lemma 2 it follows that

$$
\left\|u^{i}(r x)-p_{r}^{i}(r x)\right\|_{W^{2,2}\left(B_{1}\right)} \leq C_{n, \alpha}\left(\max _{i}\left\|\varphi^{i}\right\|_{C^{2, \alpha}}+\max _{i}\left\|u^{i}\right\|_{L^{2}}\right) r^{2+\alpha},
$$

where $C_{n, \alpha}$ is a dimensional constant.

Proof. Let us recall that $\left\|\Delta u^{i}(r x)\right\|_{L^{2}\left(B_{1}\right)} \leq C\left\|\Delta \varphi^{i}\right\|_{C^{\alpha} r^{\alpha}}$, then the statement follows from the inequalities (18), (19) and (20).

Now we are ready to prove the main theorem.

Theorem 4. Assume $\varphi^{1}, \varphi^{2} \in C^{2, \alpha}$, and $\mathscr{L}=\overline{\mathscr{L}^{0}}$ then the solution to the system $(11),\left(u^{1}, u^{2}\right)$ is $C^{2, \alpha}$-regular on $\partial \Omega_{1} \cap \partial \Omega_{2} \cap \partial \mathscr{L} \cap \Omega$, in the sense that for every $x_{0} \in \partial \Omega_{1} \cap \partial \Omega_{2} \cap \partial \mathscr{L} \cap \Omega$, there exist second order polynomials $p_{x_{0}}^{1}, p_{x_{0}}^{2}$, such that

$$
\sup _{x \in B_{r}\left(x_{0}\right)}\left|u^{i}(x)-p_{x_{0}}^{i}(x)\right| \leq C r^{2+\alpha}
$$

where the constant $C>0$ depends only on the given data.

Proof. Without loss of generality, we may assume $x_{0}=0$, and consider the following rescalings

$$
v_{r}^{i}(x)=\frac{u^{i}(r x)-p_{0}^{i}(r x)}{r^{2+\alpha}}
$$

then according to Lemma 2 and Corollary $2,\left\|v_{r}^{i}\right\|_{W^{2,2}\left(B_{1}\right)} \leq C$.

The pair $\left(v_{r}^{1}, v_{r}^{2}\right)$ solves the following system

$$
\left\{\begin{array}{l}
\min \left(-\Delta v_{r}^{1}-\frac{t r A_{0}^{1}}{r^{\alpha}}, v_{r}^{1}-v_{r}^{2}+q_{r}^{1}\right)=0 \\
\min \left(-\Delta v_{r}^{2}-\frac{\operatorname{tr} A_{0}^{2}}{r^{\alpha}}, v_{r}^{2}-v_{r}^{1}+q_{r}^{2}\right)=0 \\
\min \left(-\Delta v_{r}^{1}-\frac{t r A_{0}^{1}}{r^{\alpha}},-\Delta v_{r}^{2}-\frac{t r A_{0}^{2}}{r^{\alpha}}\right)=0,
\end{array}\right.
$$

where

$$
q_{r}^{1}(x)=\frac{p_{0}^{1}(r x)-p_{0}^{2}(r x)+\varphi^{1}(r x)}{r^{2+\alpha}}, q_{r}^{2}(x)=\frac{p_{0}^{2}(r x)-p_{0}^{1}(r x)+\varphi^{2}(r x)}{r^{2+\alpha}} .
$$

Then

$$
-\Delta v_{r}^{i}=\left\{\begin{array}{l}
\frac{\operatorname{tr} A_{0}^{i}}{r^{\alpha}}+\frac{\Delta \varphi^{i}(r x)}{r^{\alpha}} \text { if } r x \in \Omega_{i} \\
\frac{\operatorname{tr} A_{0}^{i}}{r^{\alpha}} \text { otherwise. }
\end{array}\right.
$$

We assumed that $0 \in \partial \Omega_{1} \cap \partial \Omega_{2} \cap \partial \mathscr{L}$, then $\Delta \varphi^{i}(0)=0, i=1$, 2. Hence $\left|\frac{\Delta \varphi^{1}(r x)}{r^{\alpha}}\right| \leq\left\|\varphi_{r}^{1}\right\|_{C^{2, \alpha}\left(B_{1}\right)}|x|^{\alpha}$. We know that $\left\|\Delta v_{r}^{i}\right\|_{L^{2}\left(B_{1}\right)}$ is bounded, therefore $\operatorname{tr} A_{0}^{i}=0$, and $\Delta v_{r}^{i}(x)$ is uniformly bounded. 
We have that $\left\|v_{r}^{i}\right\|_{L^{2}\left(B_{1}\right)} \leq C$ and we saw that $\left\|\Delta v_{r}^{i}\right\|_{L^{\infty}\left(B_{1}\right)} \leq\left\|\varphi^{i}\right\|_{C^{2, \alpha}\left(B_{1}\right)}$. Using the Calderon-Zygmund estimates, we conclude that $\left\|v_{r}^{i}\right\|_{C^{1, \gamma}}$ is uniformly bounded. In particular, $\left|v_{r}^{i}(x)\right| \leq C^{\prime}\left(C_{0}+\left\|\varphi^{i}\right\|_{C^{2, \alpha}\left(B_{1}\right)}\right)$ for every $x \in B_{1}$ and $r \leq \frac{1}{2}$.

Recall that we set $C_{0}=C\left(\max _{i}\left\|\Delta \varphi^{i}\right\|_{L^{\infty}}+\max _{i}\left\|u^{i}\right\|_{L^{2}\left(B_{1}\right)}\right)$, and $v_{r}^{i}(x)=\frac{u^{i}(r x)-p_{0}^{i}(r x)}{r^{2+\alpha}}$. Then we get the desired inequality

$$
\sup _{x \in B_{r}}\left|u^{i}(x)-p_{0}^{i}(x)\right| \leq C\left(\max _{i \in\{1,2\}}\left\|u^{i}\right\|_{L^{2}\left(B_{1}\right)}+\max _{i \in\{1,2\}}\left\|\varphi^{i}\right\|_{C^{2, \alpha}\left(B_{1}\right)}\right) r^{2+\alpha} .
$$

\subsection{A counterexample in case the zero-loop set has an isolated point}

Here we give a counterexample, showing that if the zero loop set has an isolated point, then the solution may not be $C^{1,1}$.

We consider the following system in $\mathbb{R}^{2}$

$$
\left\{\begin{array}{l}
\min \left(-\Delta u^{1}, u^{1}-u^{2}+\varphi\right)=0 \\
\min \left(-\Delta u^{2}, u^{2}-u^{1}+\varphi\right)=0
\end{array}\right.
$$

with $\varphi=\frac{1}{4}|x|^{2}$.

Then the difference $U=u^{1}-u^{2}$ solves the following double-obstacle problem in $\mathbb{R}^{2}$

$$
-\Delta U=\left\{\begin{array}{l}
1, \text { if } U=-\varphi \\
-1, \text { if } U=\varphi \\
0, \text { if }-\varphi<U<\varphi
\end{array}\right.
$$

and $-\Delta u^{1}=(-\Delta U)^{+}$and $-\Delta u^{2}=(\Delta U)^{+}$.

Now let us consider a function $w$ defined as follows

$$
w=\left\{\begin{array}{l}
-\frac{1}{4}|x|^{2}, \text { if } x_{1}>0, x_{2}>0 \\
\frac{1}{4}\left(x_{1}^{2}-x_{2}^{2}\right), \text { if } x_{1}<0, x_{2}>0 \\
\frac{1}{4}\left(x_{2}^{2}-x_{1}^{2}\right), \text { if } x_{1}>0, x_{2}<0 \\
\frac{1}{4}|x|^{2}, \text { if } x_{1}<0, x_{2}<0
\end{array}\right.
$$

Then $w \in C^{1,1}$ also solves the double-obstacle problem (24), therefore we choose $U \equiv w$.

Next we write $u^{1}$ explicitly in polar coordinates

$$
u^{1}(r, \theta)=\left\{\begin{array}{l}
-\frac{1}{4} r^{2}-\frac{1}{2 \pi} r^{2} \theta \cos 2 \theta-\frac{1}{2 \pi} r^{2} \ln r \sin 2 \theta, \text { if } 0<\theta \leq \frac{\pi}{2} \\
-\frac{1}{4} r^{2} \cos 2 \theta+\frac{1}{2 \pi} r^{2} \theta \cos 2 \theta+\frac{1}{2 \pi} r^{2} \ln r \sin 2 \theta, \text { otherwise, }
\end{array}\right.
$$

here the function $r^{2} \theta \cos 2 \theta+r^{2} \ln r \sin 2 \theta \in C^{1, \gamma}$ for every $0<\gamma<1$, solves the Laplace equation in $\mathbb{R}^{2} \backslash\{0\}$, but is not $C^{1,1}$ near the origin.

Therefore $u^{1}$ is a $C^{1, \gamma}$ function in the unit ball in $\mathbb{R}^{2}$ but it is not $C^{1,1}$ in the neighborhood of the origin, since $\left|\frac{\partial^{2} u^{1}}{\partial r^{2}}\right| \approx|\ln r| \rightarrow \infty$ as $r \rightarrow 0$. Moreover, $-\Delta u^{1}(r, \theta)=\chi_{\left\{0<\theta \leq \frac{\pi}{2}\right\}}=\chi_{\left\{u^{1}>0\right\}}$, provided $r>0$ is small enough.

Next we take $u^{2}(r, \theta)=u^{1}(r, \theta)-w$, then

$$
u^{2}(r, \theta)=\left\{\begin{array}{l}
-\frac{1}{2 \pi} r^{2} \theta \cos 2 \theta-\frac{1}{2 \pi} r^{2} \ln r \sin 2 \theta, \text { if } 0<\theta \leq \frac{\pi}{2} \\
-\frac{1}{2} r^{2} \cos 2 \theta+\frac{1}{2 \pi} r^{2} \theta \cos 2 \theta+\frac{1}{2 \pi} r^{2} \ln r \sin 2 \theta, \text { if } \frac{\pi}{2}<\theta \leq \pi \\
-\frac{1}{4} r^{2}-\frac{1}{4} r^{2} \cos 2 \theta+\frac{1}{2 \pi} r^{2} \theta \cos 2 \theta+\frac{1}{2 \pi} r^{2} \ln r \sin 2 \theta, \text { if } \pi<\theta \leq \frac{3 \pi}{2} \\
\frac{1}{2 \pi} r^{2} \theta \cos 2 \theta+\frac{1}{2 \pi} r^{2} \ln r \sin 2 \theta, \text { if } \frac{3 \pi}{2}<\theta \leq 2 \pi
\end{array}\right.
$$

Neither $u^{1}$ nor $u^{2}$ is a $C^{1,1}$ function. However, it is easy to see that $\left(u^{1}, u^{2}\right)$ solves (23), and it is minimal, since $\min \left(-\Delta u^{1},-\Delta u^{2}\right)=0$ a.e. 


\section{Conflict of interest statement}

I would like to confirm that there are no known conflicts of interest associated with the article "Optimal regularity in the optimal switching problem". Gohar Aleksanyan, 24 July 2015, Stockholm, Sweden.

\section{Acknowledgements}

I would like to thank Prof. John Andersson, Prof. Diogo Gomes and Prof. Henrik Shahgholian for several fruitful discussions. In particular, I would like to thank John Andersson for the idea of the proof of $C^{2, \alpha}$-regularity at the meeting points.

\section{References}

[1] John Andersson, Erik Lindgren, Henrik Shahgholian, Optimal regularity for the no-sign obstacle problem, Commun. Pure Appl. Math. 66 (2013) 245-262.

[2] Stavros A. Belbas, Suzanne M. Lenhart, Nonlinear PDEs for stochastic optimal control with switchings and impulses, Appl. Math. Optim. 14 (1986) 215-227.

[3] Filippo Cagnetti, Diogo Gomes, Hung Vinh Tran, Adjoint methods for obstacle problems and weakly coupled systems of PDE, ESAIM Control Optim. Calc. Var. 19 (2013) 754-779.

[4] Lawrence C. Evans, Avner Friedman, Optimal stochastic switching and the Dirichlet problem for the Bellman equation, Trans. Am. Math. Soc. 253 (1979) 365-389.

[5] David Gilbarg, Neil S. Trudinger, Elliptic Partial Differential Equations of Second Order, Springer-Verlag, Berlin, 2001, reprint of the 1998 edition.

[6] Diogo Gomes, António Serra, Systems of weakly coupled Hamilton-Jacobi equations with implicit obstacles, Can. J. Math. 64 (2012) 1289-1309.

[7] Suzanne M. Lenhart, Stavros A. Belbas, A system of nonlinear partial differential equations arising in the optimal control of stochastic systems with switching costs, SIAM J. Appl. Math. 43 (1983) 465-475.

[8] Arshak Petrosyan, Henrik Shahgholian, Nina Uraltseva, Regularity of Free Boundaries in Obstacle-Type Problems, American Mathematical Society, Providence, RI, 2012.

[9] Elias M. Stein, Harmonic Analysis: Real-Variable Methods, Orthogonality, and Oscillatory Integrals, Princeton University Press, Princeton, NJ, 1993. 